\title{
Delayed cardiac tamponade after open heart surgery - is supplemental CT imaging reasonable?
}

\author{
Bernhard Floerchinger*, Daniele Camboni, Simon Schopka, Philipp Kolat, Michael Hilker and Christof Schmid
}

\begin{abstract}
Background: Cardiac tamponade is a severe complication after open heart surgery. Diagnostic imaging is challenging in postoperative patients, especially if tamponade develops with subacute symptoms. Hypothesizing that delayed tamponade after open heart surgery is not sufficiently detected by transthoracic echocardiography, in this study CT scans were used as standard reference and were compared with transthoracic echocardiography imaging in patients with suspected cardiac tamponade.

Method: Twenty-five patients after open heart surgery were enrolled in this analysis. In case of suspected cardiac tamponade patients underwent both echocardiography and $C T$ imaging. Using $C T$ as standard of reference sensitivity, specificity, positive and negative predictive values of ultrasound imaging in detecting pericardial effusion/hematoma were analyzed. Clinical appearance of tamponade, need for re-intervention as well as patient outcome were monitored.
\end{abstract}

Results: In 12 cases (44\%) tamponade necessitated surgical re-intervention. Most common symptoms were deterioration of hemodynamic status and dyspnea. Sensitivity, specificity, positive and negative predictive values of echocardiography were 75\%,64\%, 75\%, and 64\% for detecting pericardial effusion, and 33\%, 83\%, 50, and 71\% for pericardial hematoma, respectively. In-hospital mortality of the re-intervention group was $50 \%$.

Conclusion: Diagnostic accuracy of transthoracic echocardiography is limited in patients after open heart surgery. Suplemental CT imaging provides rapid diagnostic reliability in patients with delayed cardiac tamponade.

Keywords: Cardiac tamponade, Computer tomography, Transthoracic echocardiography

\section{Background}

Pericardial effusion is common in patients after open heart surgery due to postoperative bleeding or postcardiotomy syndrome [1]. Hemodynamic relevant effusion following cardiac surgery leading to tamponade is a potentially lifetreatening condition, therefore rapid diagnosis and therapy is essential [2]. Echocardiography is the standard tool to fix diagnosis and assess compromised atrial and ventricular (un)loading. Featuring excellent sensitivity and specifity in patients without previous open heart surgery, postoperative imaging with transthoracic echocardiography (TTE) is affected by modified anatomy and other issues, i.e. mechanical ventilation. Correspondingly, detection of pericardial effusion in postoperative patients can be less sensitive, and transoesophageal assessment is required [3]. Transoesophageal echocardiography (TEE) is fast and easy

*Correspondence: bernhard.floerchinger@ukr.de

Department Cardiothoracic Surgery, University Medical Center Regensburg, 93053, Regensburg, Germany available in intensive care settings with ventilated patients after cardiac surgery, but represents a semi-invasive diagnostic tool, uncomfortable for spontanous breathing and not-sedated patients. Even if TEE has a high safety profile with low complication rates, an alternative diagnostic tool such as computer tomography $(\mathrm{CT})$ imaging is a valuable option in patients not sufficiently assessed by TTE [4].

Hypothesizing that delayed and subacute cardiac tamponade is not detected sufficiently by transthoracic echocardiography in patients after open heart surgery, we evaluated patients with suspected non-acute pericardial effusion in regard to hemodynamic tamponade and need for intervention. Diagnostic value of transthoracic echocardiography was compared to thoracic CT scans in patients with suspected pericardial effusion postoperatively. Symptoms leading to cardiac tamponade as well as outcome of patients undergoing re-intervention are reported.

\section{()

(c) 2013 Floerchinger et al.; licensee BioMed Central Ltd. This is an Open Access article distributed under the terms of the Creative Commons Attribution License (http://creativecommons.org/licenses/by/2.0), which permits unrestricted use, distribution, and reproduction in any medium, provided the original work is properly cited. 


\section{Method}

\section{Patients}

From October 2011 until November 2012, all patients undergoing transthoracic echocardiography and thoracic CT scans due to suspected pericardial effusion after open heart surgery were included in this study. Written informed consent for diagnostic imaging was obtained, but requirement of individual patient consent was waived due to the retrospective design of the study in accordance with rules of the institutional ethical committee of University Regensburg. Patients with acute cardiac tamponade requiring immediate resternotomy in the operating room or on intensive care unit were not included in this analysis. Twenty five patients (mean $67 \pm 11$ years, range 5085 years) were enrolled retrospectively. Baseline characteristics and initial cardiac surgery are shown in Table 1.

Intraoperatively, pericardium was adapted by two single sutures in case of routine cardiac surgery procedures (coronary artery bypass grafting, aortic/mitral valve replacement/repair). Pericardium was not adapted after heart transplantation, ventricular assist device implantation, aortic and redo-surgery. Two chest tubes were placed in the pericardium with additional tubes for pleural drainage, if necessary. With drainage volume less than $400 \mathrm{~mL}$ chest tubes were removed on first postoperative day, otherwise tubes were kept in pericardial space until drain volume felt below $200 \mathrm{~mL}$ per 24 hours.

Diagnostic imaging was initiated in case of new-onset symptoms indicating pericardial effusion or tamponade, respectively: collapse, syncope, dyspnea, deterioration of circulatory status (i.e. new-onset/increasing need for

Table 1 Baseline characteristics of patients enrolled

\begin{tabular}{lll}
\hline & $\mathbf{N}$ & $\%$ \\
\hline Patients & 25 & 100 \\
\hline Gender & 19 & 76 \\
\hline Male & 6 & 24 \\
\hline Female & $67 \pm 11$ years & \\
\hline Median age & $(50-85$ years $)$ & \\
\hline Previous cardiac surgery & $21 \pm 42$ days & \\
\hline CABG & $(1-209$ days $)$ & \\
\hline AVR & & 60 \\
\hline MVR & $\mathrm{n}=15$ & 32 \\
\hline Ascending aortic & $\mathrm{n}=8$ & 12 \\
\hline Replacement & $\mathrm{n}=3$ & 12 \\
\hline Other (HTX, VAD, pericardectomy) & $\mathrm{n}=3$ & \\
\hline Combined & $\mathrm{n}=3$ & 12 \\
\hline Redo surgery & $\mathrm{n}=6$ & 24 \\
\hline
\end{tabular}

catecholamine support, increased central venous pressure) without other underlying pathology evident.

Patients underwent surgical re-intervention when pericardial effusion/tamponade were considered hemodynamically relevant. Hemodynamic relevance was determined as compromised (un) loading of left/right atrium/ventricle evident and correlating with clinical symptoms (see above). Suspected pericardial tamponade was confirmed, if more than $300 \mathrm{~mL}$ pericardial fluid/ thrombus formation were removed as well as hemodynamic status immediately stabilized (decreasing catecholamine need and central venous pressure, increasing mean arterial pressure) following pericardial relieving manoeuvre (pericardiocentesis, resternotomy).

\section{Echocardiography}

Transthoracic echocardiography was performed with a Philips CX50 CompactXtreme or a Philips iE33 xMatrix echo system (Royal Philips Electronics N.V., Amsterdam, Netherlands) by physicians or cardiac surgeons experienced with more than 1000 documented transthoracic echocardiography procedures. Examination included parasternal long and short axis, apical four/five chamber view, as well as subxiphoidal view. Maximum extent of pericardial effusion was recorded, left/right atrium and ventricle were assessed in regard to unaffected/ compromised (un)loading and myocardial function/contractility. Finally, operators classified echocardiography imaging sufficient or not-sufficient for assessment.

\section{Computertomography}

Thoracic CT scans were performed with a 16-slice CT canner (Siemens Somatom Sensation 16, Siemens Healthcare AG, Erlangen, Germany). Reconstruction slice thickness was 1-5 mm. In case of suspected pericardial hemorrhage a volume of $120 \mathrm{~mL}$ contrast medium (Omnipaque, GE Healthcare, Waukesha, WI, USA) was infused intravenously (infusion rate $\sim 4 \mathrm{~mL} / \mathrm{sec}$ ). For this retrospective analysis, CT findings were assessed by a radiologist unaware of echocardiography results.

\section{Statistics}

Statistical analysis was performed with GraphPad Prism 5.03 (GraphPad Inc., San Diego, CA, USA) In descripitive analysis continuous variables are given as mean values with standard deviation. Sensitivity, specificity, positive and negative predictive values were calculated using a Fisher's exact test. Differences of continuous variables were compared by Student's t-test and were considered statistically significant with probability of 0.05 or less.

\section{Results}

Twenty-five patients were enrolled in this analysis; two patients were enrolled repeatedly due to recurring tamponade symptoms. Mean time point of diagnostic 
imaging for pericardial effusion was $21 \pm 42$ postoperative days in all cases. Five patients had been readmitted to hospital, 20 patients were screened during postoperative stay. Eleven patients were on normal ward, 14 patients were on intensive $(n=12)$ / intermediate $(n=2)$ care unit. Two patients were readmitted to intensive care due to hemodynamic and respiratory collapse, respectively.

Reasons for initiating diagnostic imaging were deterioration of hemodynamic status in 16 pts. (increasing/newonset need for catecholamine support (14 pts.), increasing serum lactate level (1 pt.), collapse (1 pt.), low-cardiac-output (3 pts.), right-heart-failure (1 pt.)), dyspnea in 7 patients, and suspected wound infection/sternum dehiscence in 7 patients, respectively. One patient suffered from recurring collapse leading to readmission, another from new-onset acute renal failure. Finally, five patients had a concomitant hematothorax. In total, 14 patients required catecholamine therapy (new-onset need in 4 patients). Inotropic ( $\mathrm{n}=8$ pts., overall: dobutamine $1.875 \pm 0.177$ $\mu \mathrm{g} / \mathrm{KG} / \mathrm{min}$ or suprarenine $0.076 \pm 0.033 \mu \mathrm{g} / \mathrm{KG} / \mathrm{min}$, respectively) and vasopressor support $(\mathrm{n}=11$ pts., overall: $0.093 \pm 0.1 \mu \mathrm{g} / \mathrm{KG} / \mathrm{min}$ ) was comparable in patients with and without subsequent intervention $(\mathrm{p}=\mathrm{n}$.s.). Sixteen patients were breathing spontaneously when undergoing CT imaging. Nine patients were on ventilation therapy, 5 patients had been intubated due to respiratory collapse prior to diagnostic imaging.

\section{Echocardiography vs. CT}

Transthoracic echo and CT scans matched in detecting pericardial effusion in 12 patients (44\%). Mean diameters of detected pericardial effusion were equivalent ( $C T$ vs. TTE: $17 \pm 11$ vs. $18 \pm 8 \mathrm{~mm}, \mathrm{p}=$ n.s.) (Figure $1 \mathrm{~A}, \mathrm{~B}$ ). In 8 patients (30\%) TTE and CT results were not consistent. Pericardial effusion was not detected positively in 4 patients by either TTE or CT, respectively. Another 7 patients (26\%) did not have pericardial fluid in both screening methods consistently.
Using CT scan results as standard of reference sensitivity of echocardiography for pericardial effusion was $75 \%$, specificity was $64 \%$. Positive and negative predictive values were $75 \%$ and $64 \%$, respectively.

CT scans revealed pericardial hematoma in 9 patients (mean $54 \pm 33 \mathrm{~mm}$, range $19-130 \mathrm{~mm}$ ), mainly located in the retrosternal mediastinal space $(8 \mathrm{pts}$.). One patient featured a $102 \times 95 \mathrm{~mm}$ hematoma in the right atrial pericardium (Figure 2). Echocardiography sensitivity for detecting hematoma was $33 \%$ and specificity $83 \%$. Positive and negative predictive values for hematoma were $50 \%$ and $71 \%$, respectively (Table 2 ). In 7 patients, operators classified transthoracic echocardiography not sufficient to evaluate tamponade.

In 12 cases $(\sim 4 \%)$ pericardial effusion and hematoma were deemed as hemodynamically relevant with need for surgical re-intervention. Mean time point of re-intervention was postoperative day $20 \pm 27$ (range 1-95d).

Ten patients underwent resternotomy, in 2 cases tamponade was relieved via subxiphoid pericardiotomy. Evidence for cardiac tamponade was confirmed in 11 cases. All procedures were performed without cardiopulmonary bypass support.

In fifteen cases (56\%) no intervention was done, two patients received antiinflammatory medication (steroids and non-steroidal antirheumatic drugs), due to postcardiotomy syndrome. In-hospital-mortality was significantly increased in patients requiring intervention in contrast to patients without intervention (50\% (6 pts.) vs. $7 \%$ (1 pt.), $\mathrm{p}=0.009)$. One patient of the no-intervention group deceased after emergency CABG due to severe lowcardiac-output leading to multi-organ-failure on postoperative day 4 . In the intervention group 4 patients died due to multi-organ-failure was on mean postoperative day $99 \pm 115$ after initial surgery. Two patients died due to respiratory and circulatory failure (on postoperative day 105 and 18), respectively.
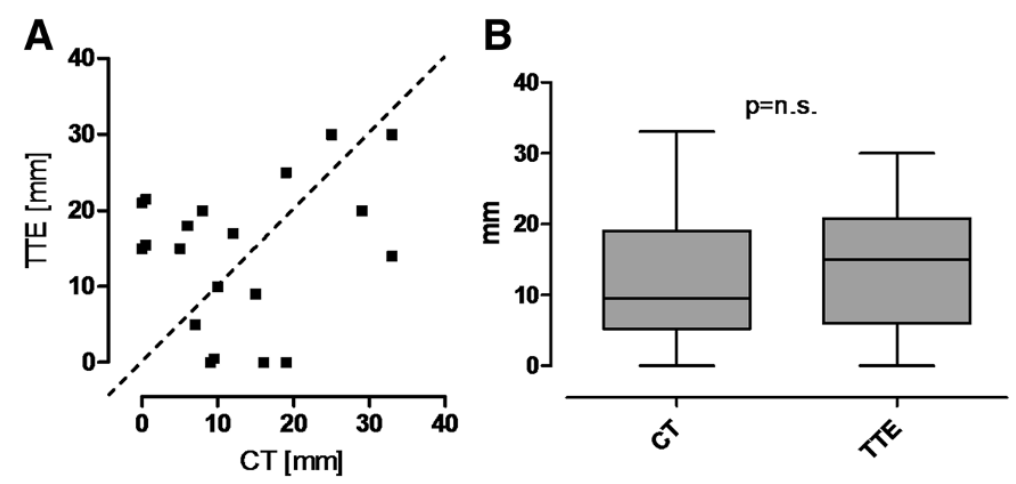

Figure 1 Visualization of corresponding (A) and mean (B) CT and echocardiography values of maximum pericardial effusion [mm] detected. 

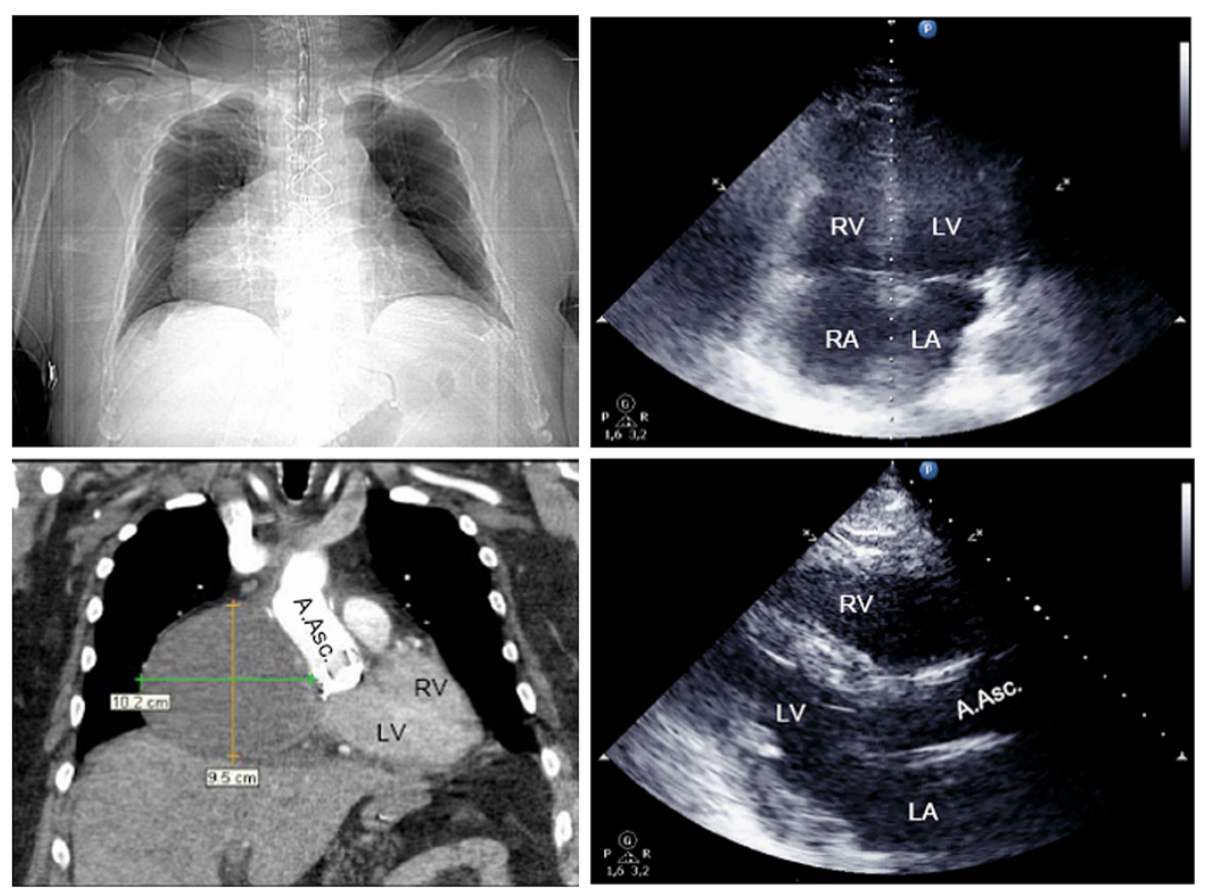

Figure 2 Patient with cardiac tamponade on day 15 after ascending aortic replacement. Right atrial hematoma $(102 \times 95 \mathrm{~mm})$ not detected by transthoracic echocardiography (B-mode: apical 4-chamber-view right above, parasternal long axis right below). Thoracic CT-imaging with contrast medium, arterial phase (topography scan left above, coronary view, soft tissue window below left). Diagnostic imaging initiated after collapse necessitating cardiopulmonary resuscitation. LV left ventricle, RV right ventricle, LA left atrium, RA right atrium, A.Asc. ascending aorta.

\section{Discussion}

Echocardiography is fundamental for imaging the heart and pericardial structures, since fast available and applicable with adequate sensitivity/specificity. Patients after open heart surgery represent a separate population with pericardial effusion up to more than $60 \%$ [1]. Effusion leading to cardiac tamponade is a potentially lifetreatening condition, therefore rapid and reliable diagnostic imaging is essential in patients with tamponade suspected. Especially subacute cardiac tamponade represents a challenging entity to detect with delayed evidence of clinical symptoms. Classic symptoms, such as hypotension, tachycardia, pulsus paradoxus, increased central venous pressure, as well as low urine output can be masked after cardiac surgery or alleviated in case of slowly increasing effusion [5-7]. Of note, in this cohort

Table 2 Sensitivity, specificity, positive and negative predictive values for pericardial effusion and hematoma of transthoracic echocardiography using computer tomography scans as standard of reference

\begin{tabular}{lll}
\hline & Pericardial effusion & Hematoma \\
\hline Sensitivity & $75 \%$ & $33 \%$ \\
\hline Specificity & $64 \%$ & $83 \%$ \\
\hline Positive predictive value & $75 \%$ & $50 \%$ \\
\hline Negative predictive value & $64 \%$ & $71 \%$ \\
\hline
\end{tabular}

Calculation by Fisher's exact test. interventions were done on mean postoperative day 20 with only 3 patients within 6 days after initial surgery. This indicates incidence of delayed cardiac tamponade not being affected by late removal of chest tubes. As described previously, prolonged chest tube drainage does not impact incidence of pericardial effusion $[8,9]$. Due to patients' discomfort, the authors' institutional policy implicates tube removal on postoperative day one, if drain volume is less than $400 \mathrm{~mL}$ without increased incidence of pericardial effusion evident.

Also, patients with and without tamponade featured comparable inotropic and vasopressor support. Therefore, prolonged/new-onset need for catecholamine support cannot be used as sensitive indicator for delayed tamponade, since unspecific hemodynamic decline is caused also by other reasons, such as infection or respiratory issues.

Moreover, majority of pericardial effusion after cardiac surgery is not circumferential but localized, then even more challenging to be detected [7]. Localized intrapericardial effusion and clots after cardiac surgery are frequently placed in the posterior pericardium or near the right atrium and ventricle with limited assessability via TTE [10]. Benefits of TTE are easy availability, high patient comfort, and comprehensive dynamic information regarding myocardial and valve function as well as intravascular volume status, but conflicting sensibility 
and specificity in imaging pericardial effusion have been reported $[11,12]$. In this analysis sensitivity and specificity were poor for both localized hematoma and circumferential effusion. Only 2 of 4 patients with pericardial hematoma requiring resternotomy were detected correctly. Ultrasound imaging is not only operator-dependent but also considerably affected by postoperative issues, such as altered anatomy, mechanical ventilation, as well as drains and trapped air in thoracic cavity, leading to notsatisfying imaging.

Correspondingly, in 7 cases TTE was classified not sufficient for assessing tamponade with additional imaging required. In face of increasing use of intrapericardial VAD systems this issue will be aggravated. In the analyzed cohort only 1 patient with LVAD with recurring tamponade was included. Diastolic collapse of right atrium and ventricle has been postulated as reliable sign for cardiac tamponade in echocardiography $[13,14]$. Interestingly, in patients enrolled, no diastolic collapse was detected; merely in one patient with right atrial hematoma impression of right atrium was evident. This could be caused by tamponade developing gradually without permanent diastolic collapse of the right heart. Assessability may be improved by using transoesphageal echocardiography (TEE). TEE featured high efficacy to ascertain hematoma in a recent $23 \mathrm{ICU}$ patient analysis with surgically proven hematoma following cardiac surgery [15]. Even TEE is well-tolerated also in critically ill patients with few complications, our institution favours CT imaging for clarifying suspected tamponade, as it features several benefits. CT scans offer objective and not operator-dependent static imaging of thoracic anatomy. Also, other issues such as suspected sternum infection or dyspnea, or hemorrhage (evident in $56 \%$ of enrolled patients) are approached by CT scans without additional procedure.

This is particulary reasonable in hemodynamically stable patients not on ICU and at postoperative day 7 or more (in this cohort $52 \%$ and $64 \%$, respectively) to gain detailed information about thoracic anatomy before resternotomy. Otherwise, contrast medium supported $\mathrm{CT}$ imaging features the risk of renal impairment and allergy-like reactions [16]. Despite this risk for imaging-related complications, a non-acute pericardial tamponade is displayed effectively via suplemental CT imaging with high sensitivity and specificity, as frequently reported after blunt chest trauma [17-19]. Therefore, CT imaging represents a valuable option to evaluate patients with suspicion of cardiac tamponade after open heart surgery.

\section{Conclusion}

Diagnostic accuracy of transthoracic echocardiography is limited in patients after open heart surgery, since sensitivity and specificity for detection of cardiac tamponade are limited. Therefore, CT imaging is reasonable for supplemental diagnostic imaging to assess suspected delayed cardiac tamponade in high-risk patients after open heart surgery.

\section{Competing interest}

All authors declare no conflict of interest regarding this manuscript. Also, all authors have full access and controls of data used in this manuscript, and agree to allow the journal to review, if requested.

Received: 5 March 2013 Accepted: 20 June 2013

Published: 24 June 2013

\section{References}

1. Pepi M, Muratori $M$, Barbier $P$, et al: Pericardial effusion after cardiac surgery: incidence, site, size, and haemodynamic consequences. Br Heart J 1994, 72:327-31.

2. Bodson L, Bouferrache K, Vieillard-Baron A: Cardiac Tamponade. Curr Opin Crit Care 2011, 17:416-24.

3. Kochar GS, Jacobs LE, Kotler MN: Right atrial compression in postoperative cardiac patients: Detection by transoesophageal echocardiography. J Am Coll Cardiol 1990, 19:511-16.

4. Hilberath J, Oakes DA, Sherman SK, et al: Safety of transoesophageal echocardiography. J Am Soc Echocardiography 2010, 23:1115-27. R.

5. Russo AM, O'Connor WH, Waxman HL: Atypical presentations and echocardiographic findings in patients with cardiac tamponade occurring early and late after cardiac surgery. Chest 1993, 104:71-8.

6. Grecu L: Cardiac tamponade. Int Anesthesiol Clin 2012, 50:59-77.

7. Chuttani K, Tischler MD, Pandian NG: Diagnosis of cardiac tamponade after cardiac surgery: relative value of clinical, echocardiographic, and hemodynamic signs. Am Heart J 1994, 127:913-8.

8. Smulders YM, Wiepking ME, Moulijn AC, et al: How soon drainage tubes be removed after cardiac operations. Ann Thorac Surg 1989, 48:540-3.

9. Gercekoglou H, Aydin NB, Dagdeviren B, et al: Effect of timing of chest tube removal on development of pericardial effusion following cardiac surgery. J Card Surg 2003, 18:217-24.

10. Beppu S, Tanaka N, Nakatani S, et al: Pericardial clot after open heart surgery: its specific localization and haemodynamics. Eur Heart J 1993, $14: 230-234$

11. Gunteroth WG: Sensitivity and specificity of echocardiographic evidence of tamponade: implications for ventricular interdependence and pulsus paradoxus. Pediatr Cardiol 2007, 28:358-362

12. Ristic $A D$, Seferovic $P M$, Maisch B: Management of pericardial effusion: the role of echocardiography in establishing the indications and the selection of the approach for drainage. Herz 2005, 30:144-50.

13. Armstrong WF, Scbih BF, Helper D, et al: Diastolic collapse of the right ventricle with cardiac tamponde: an echocardiogaphic study. Circulation 1982, 65:1491-6.

14. Gillam LD, Coyer DE, Gibson TC, et al: Hemdynamic compression of right atrium: a new echocardiographic sign of cardiac tamponade. Circulation 1983, 68:294-301.

15. Grumann A, Baretto L, Dugard A, et al: Localized cardiac tamponade after open-heart surgery. Ann Thorac Cardiovasc Surg 2012. Epub ahead of print

16. Schönenberger $\mathrm{E}$, Mühler $\mathrm{M}$, Dewey $\mathrm{M}$ : Complication due to contrast agent administration. Internist 2010, 51:1516-24.

17. Restrepo CS, Gutierrez FR, Marmol-Velez JA, et al: Imaging patients with cardiac trauma. RadioGraphics 2012, 32:633-649.

18. Hermens JA, Wajon EM, Grandjean JG, et al: Delayed cardiac tamponade in a patient with previous minor blunt chest trauma. Int J Cardiol 2009, 131:e124-e126.

19. Levin S, Maldonado I, Rehm C, et al: Cardiac tamponade without pericardial effusion after blunt chest trauma. Am Heart J 1996, 131:198-200.

\section{doi:10.1186/1749-8090-8-158}

Cite this article as: Floerchinger et al:: Delayed cardiac tamponade after open heart surgery - is supplemental CT imaging reasonable?. Journal of Cardiothoracic Surgery 2013 8:158. 